\title{
Noninvasive Sensing Techniques 2012
}

\author{
Francesco Soldovieri, ${ }^{1}$ Jean Dumoulin, ${ }^{2}$ Nicola Masini, ${ }^{3}$ \\ Raffaele Solimene, ${ }^{4}$ and Erica Utsi ${ }^{5}$
}

${ }^{1}$ Istituto per il Rilevamento Elettromagnetico dell'Ambiente, Consiglio Nazionale delle Ricerche, Via Diocleziano 328, 80124 Napoli, Italy

${ }^{2}$ Monitoring, Assessment and Computational Science Department (MACS), French Institute of Science and Technology for Transport, Development and Networks (IFSTTAR), Route de Bouaye, CS4, 44344 Bouguenais, France

${ }^{3}$ Istituto per i Beni Archeologici e Monumentali, Consiglio Nazionale delle Ricerche, C. da S. Loja, 85050 Tito Scalo, Italy

${ }^{4}$ Dipartimento di Ingegneria dell'Informazione, Seconda Università di Napoli, Via Roma 29, 81031 Aversa, Italy

${ }^{5}$ Utsi Electronics Ltd., Cambridge CB22 7NZ, UK

Correspondence should be addressed to Francesco Soldovieri, soldovieri.f@irea.cnr.it

Received 5 August 2012; Accepted 5 August 2012

Copyright (C) 2012 Francesco Soldovieri et al. This is an open access article distributed under the Creative Commons Attribution License, which permits unrestricted use, distribution, and reproduction in any medium, provided the original work is properly cited.

The preservation of both the cultural heritage and modern civil and critical infrastructures is a matter of transnational concern worldwide not only in areas subject to natural disasters but also, for example, in the name of health and safety where such structures continue in regular use. Structural preservation relies uniquely on our ability to monitor accurately and form reliable diagnoses of the current condition of built structures both in terms of their material composition and any deterioration to date. These same diagnostics give us the capability to monitor over time even where such structures are not apparently at unusual levels of risk. They also allow us to manage any necessary work before and after major crisis events such as, for example, earthquakes, landslides, or floods.

This special issue of the International Journal of Geophysics focuses on noninvasive sensing techniques which form the principal method of monitoring cultural heritage and civil engineering infrastructures in order to define its current condition and ensure its preservation now and into the future.

The papers included in this issue fall into two main categories.

The first of these is the development of innovative analysis methods in order to improve the understanding and interpretation of the results of noninvasive investigations with the aim of improving the diagnostic abilities. Three of the ten papers form this category.
In their first contribution, A. D'Alterio and R. Solimene report on a new method regarding the possibility to estimate the electromagnetic parameters of a structure starting from ground penetrating radar (GPR) measurements. They extend their previous work on characterising the signal responses from the ground surface to include antenna characteristics and achieve this by taking measurements from multiple offset positions and using multiple polarisation configurations.

In their second contribution, R. Solimene and A. D'Alterio develop a method of highlighting targets embedded within a masonry wall by eliminating background clutter from GPR traces. They do this by developing a windowing strategy based on an entropy measures of temporal traces "similarity." As a result, the visibility of the targeted objects is strongly improved.

The third paper in this category deals with the development of methods of data interpretation. R. Persico demonstrate the application of a data processing method based on the Born approximation to GPR data in order to improve the detectability and geometry estimation of buried targets with both electrical and magnetic characteristics. The results are compared with the more traditional processing method of migration.

The second main category and the one into which the majority of the papers fall is that of practical applications of noninvasive investigative techniques to a wide variety of 
historical monuments and other natural resources. The techniques used in these studies are extremely wide ranging and are a good illustration of the resources available to evaluate heritage buildings, culturally sensitive structures as well as their environmental contexts. They include electromagnetic induction (EMI), electrical resistivity tomography (ERT), ground penetrating radar (GPR), magnetic susceptibility, synthetic aperture radar interferometry, the use of highresolution satellite imaging, sonic, and mechanical methods.

A. Novo et al. apply a suite of geophysical techniques (notably EMI and ERT) to the investigation of an ancient mound, Khirbat Faynan, in Southern Jordan in order to define the archaeological remains. Although they did not apply GPR due to the surface conditions, they include this in their appraisal of the work done to date and their recommendations for future potential investigations.

Turning to a more environmental context, M. A. ElSadek et al. used a range of primarily magnetic methods to investigate and identify the locations of beach-alluvial deposits of the Koam Mashaal area of East Rosetta in Egypt, demonstrating the usefulness of these methods for this type of mapping investigation.

Ground penetrating radar was used by J. N. Malik et al. within an earthquake zone, the Hajipur Fault in North West Himalaya, India, to identify a suitable trenching site and to investigate the geometry of the fault plane. A series of 2dimensional data profiles were combined to form an image of the fault plane in 3 dimensions.

D. Tapete and F. Cigna applied a procedure based on the application of Persistent Scatterer Interferometry to Synthetic Aperture Radar repeated passages for a multispatial/temporal hazard assessment of cultural heritage sites in Tuscany, in Italy, in areas critically affected by landslides. From their analyses at these sites, they developed a rapid mapping and deformation analysis intended to aid in preservation of critically endangered historical sites.

The ancient city of Ur in Mesopotamia (modern day Iraq) was investigated by G. Di Giacomo and G. Scardozzi using multitemporal high-resolution satellite images in order to analyse the structure and composition of this world heritage site. The results show the correlation of data from excavation and remote monitoring over a lengthy time period and point the way to a potential method for a longterm monitoring.

Moving into a more modern context, albeit concerning buildings of cultural and historical interest, A. Guida et al. applied ultrasound and mechanical investigation techniques to a historic building composed of reinforced concrete mixed with stone. They found that combining the results of the two methods provided an efficient method of evaluating the building and a basis on which to preserve and transform it for future use (ultimately the best method of ensuring future preservation).

Finally, in a category of its own, one paper deals with the application of developing technology. Vivaldi antennas are not new but are more traditionally applied to microwave applications. G. Ruvio et al. applied the use of Vivaldi antennas to monitor the water content of polyvinyl chloride pipes and communicate this data to an external gateway. They illustrate the operational characteristics of these antennas with a number of relevant tests.

The common theme of all of these papers is the application of a wide range of geophysical techniques to the problems of assessing and monitoring structures of historical, cultural and social importance without causing further damage to the structures themselves or triggering damaging external environmental effects. 

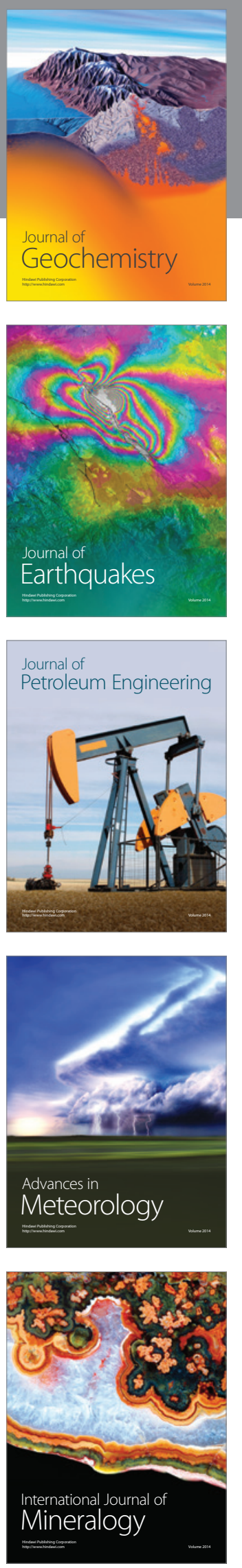
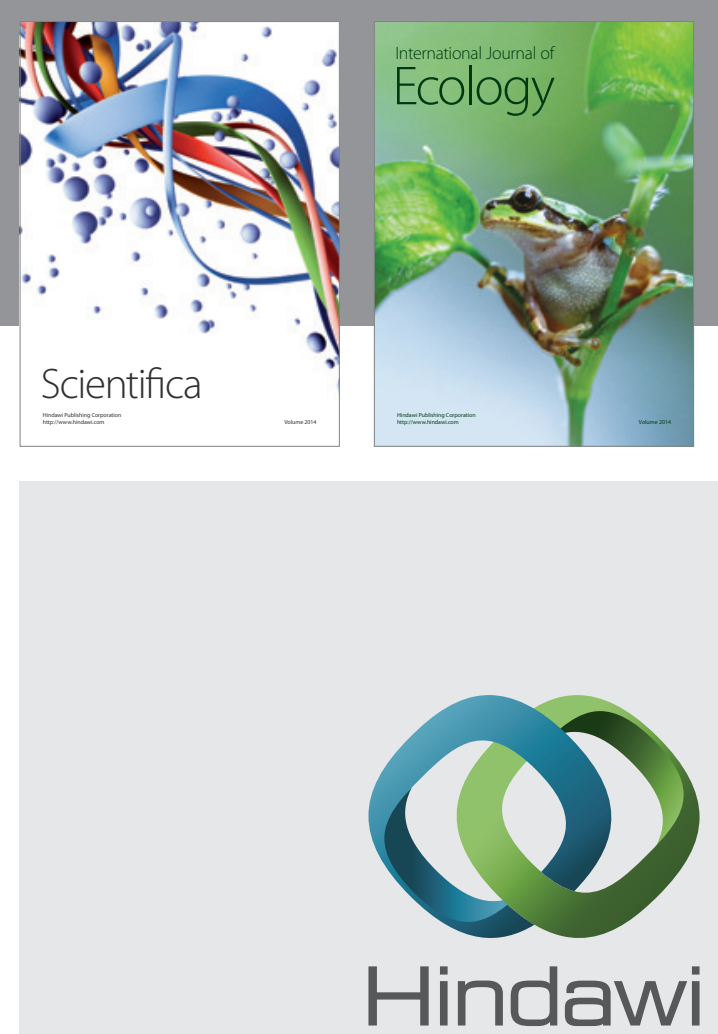

Submit your manuscripts at http://www.hindawi.com
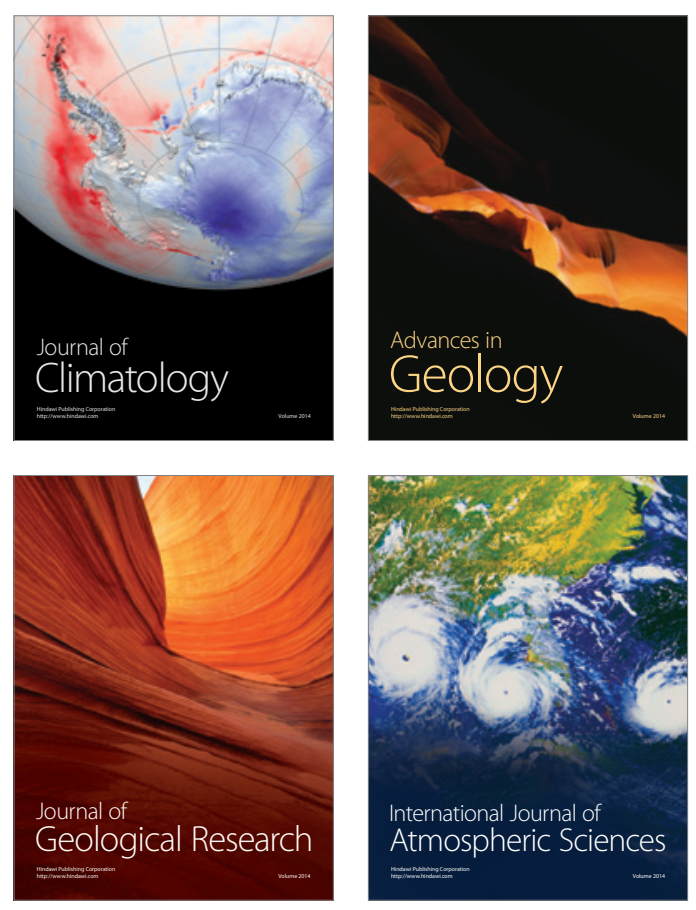
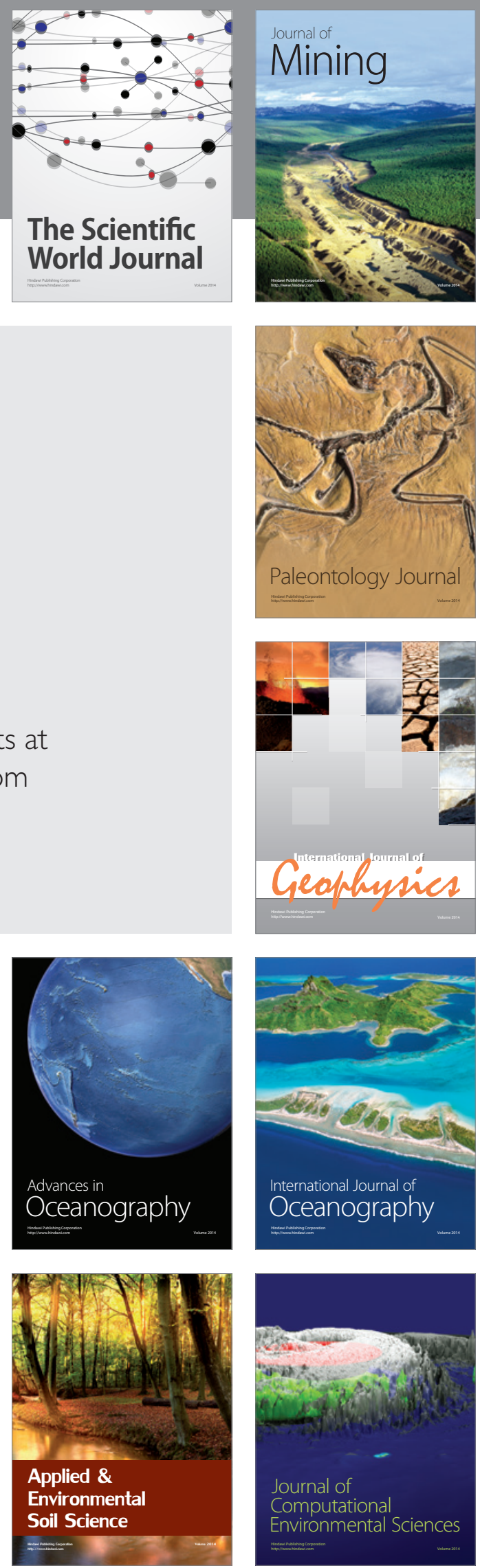